\title{
Differential Activation in Somatosensory Cortex for Different Discrimination Tasks
}

\author{
Christoph Braun, ${ }^{1}$ Renate Schweizer, ${ }^{1}$ Thomas Elbert, ${ }^{2}$ Niels Birbaumer, ${ }^{1}$ and Edward Taub ${ }^{3,4}$ \\ 1/nstitute of Medical Psychology and Behavioral Neurobiology, University of Tübingen, D-72074 Tübingen, Germany, \\ 2Department of Psychology, University of Konstanz, D-78457 Konstanz, Germany, ${ }^{3}$ Department of Psychology, University \\ of Alabama at Birmingham, Birmingham, Alabama 35294, and ${ }^{4}$ Birmingham Veterans Affairs Medical Center, Birmingham, \\ Alabama 35233
}

\begin{abstract}
Maps of the body surface in somatosensory cortex have been shown to be highly plastic, altering their configuration in response to changes in use of body parts. The current study investigated alterations in the functional organization of the human somatosensory cortex resulting from massed practice. Over a period of 4 weeks, subjects were given synchronous tactile stimulation of thumb (D1) and little finger (D5) for $1 \mathrm{hr} / \mathrm{d}$. They had to identify the orientation of the stimuli. Neuroelectric source localization based on high-resolution EEG revealed that, when subjects received passive tactile stimulation of D1 or D5, the representations of the fingers in primary somatosensory cortex were closer together after training than before. There was also an apparently correlative tendency to anomalously mislocalize near-threshold tactile stimuli equally to the distant
\end{abstract}

finger costimulated during training rather than preferentially to the finger nearest to the finger stimulated in a post-training test. However, when the stimulus discrimination had to be made, neuroelectric source imaging revealed that the digital representations of D1 and D5 were further apart after training than before. Thus, the same series of prolonged repetitive stimulations produced two different opposite effects on the spatial relationship of the cortical representations of the digits, suggesting that differential activation in the same region of somatosensory cortex is specific to different tasks.

Key words: cortical reorganization; plasticity; learning; somatosensory evoked potentials; dipole source analysis; tactile sensation threshold; human
In recent years, numerous experiments have demonstrated that the adult human nervous system retains the ability to reorganize itself in response to both injury (Elbert et al., 1994; Flor et al., 1995; Mühlnickel et al., 1998) and increased use of a body part (Elbert et al., 1995, 1997; Kaas, 1995; Buonomano and Merzenich, 1998; Sterr et al., 1998a). The latter phenomenon, frequently referred to as use-dependent cortical reorganization (Jenkins et al., 1990; Recanzone et al., 1992), has given rise to the concept that the homuncular maps of the body in the somatosensory system of the brain are quite flexible. The suggestion is that the maps are being continually reconfigured to reflect the amount $\mathrm{tk}$;2of use and consequent behaviorally relevant sensory inflow from different parts of the body. This ongoing process has been explained in terms of a continuous competition between body parts for amount of representation in cortical space (Merzenich et al., 1984).

Cortical reorganization may also be induced by manipulating the temporal correlation of two sensory events. Clark and coworkers (Clark et al., 1988; Allard et al., 1991) showed in monkeys that synchronous input from two adjacent fingers produced by surgical fusion resulted in a fusion of the receptive fields of those two fingers in somatosensory cortex. Similar results were reported for humans with congenital webbed fingers (Mogilner et

Received July 6, 1999; revised Sept. 29, 1999; accepted Sept. 30, 1999.

This research was supported by the Deutsche Forschungsgemeinschaft and Grant B95-975R from the Rehabilitation Research and Development Service, United States Department of Veterans Affairs. We thank W. Kern for excellent technical support and Anne Wilms for excellent experimental assistance.

Correspondence should be addressed to Christoph Braun, MEG-Center, OtfriedMüller-Strasse 47, 72076 Tübingen, Germany. E-mail: christoph.braun@unituebingen.de.

Copyright (C) 1999 Society for Neuroscience $\quad 0270-6474 / 99 / 200446-05 \$ 15.00 / 0$ al., 1993). In addition, simultaneous stimulation of separated body sites was found to lead to the emergence of a new atypical cortical zone jointly representing those sites (Wang et al., 1995), and blind braille readers who read with three fingers of each hand simultaneously were found to have a topographically disordered representation of the digits (Sterr et al., 1998a,b).

The present study was performed to determine whether prolonged simultaneous stimulation of two fingers on the same hand combined with a tactile discrimination task could be shown to produce both a use-dependent increase in cortical representation [as predicted by Jenkins et al. (1990), Recanzone et al. (1992), and Elbert et al. (1995)] and a simultaneity-induced disarrangement or fusion in cortical representation [as predicted by Wang et al. (1995) and Sterr et al. (1998b)]. It was expected that the outcome would depend on the nature of stimulus and task conditions used during the mapping procedure.

The discrimination task had two salient aspects: (1) stimuli were directional arrows applied to the skin, and subjects had to identify their orientation; and 2) stimulation of thumb (D1) and little finger (D5) was simultaneous $80 \%$ of the time. Highresolution EEG recordings were performed during the discrimination procedure, and the location of the cortical representation of the two digits was determined by analysis of the responses evoked in the remaining $20 \%$ of trials when stimulation was provided only to D1 or D5. In addition, EEG was also recorded in a pretraining and post-training session during which D1 and D5 were stimulated and the subject did not perform a discrimination task. We also tested whether simultaneity-induced fusion in cortical representation would result in psychophysically measurable perceptual changes. 


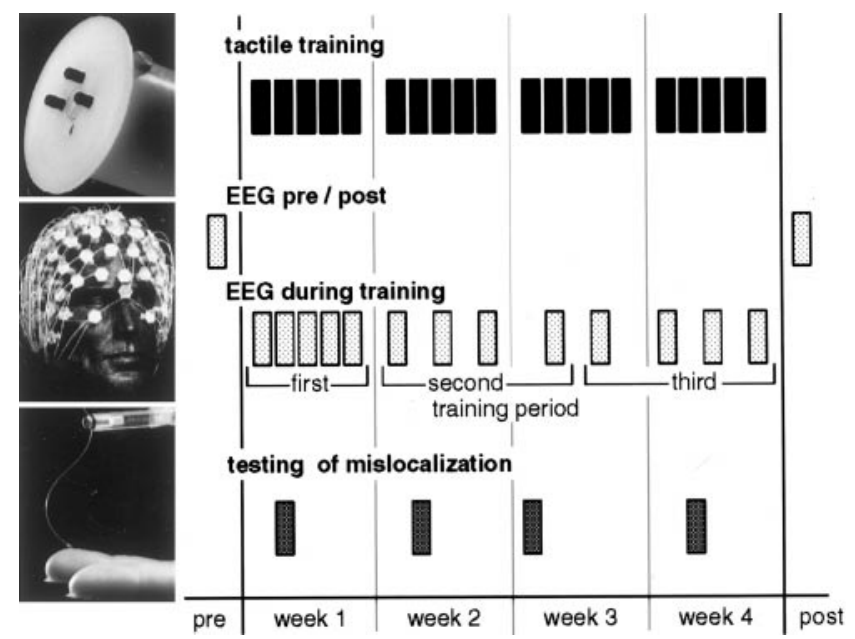

Figure 1. The timing of tactile discrimination training (top row), EEGrecording (middle row), and mislocalization testing (bottom row) across the experimental sessions.

\section{MATERIALS AND METHODS}

Subjects. The subjects were five right-handed [according to the Edinburgh inventory (Oldfield, 1971)] volunteers (three women) with a mean age of 27 (range of 22-34). All subjects signed informed consent after receiving a detailed explanation of the procedure of the study.

Tactile discrimination training and stimulation. Tactile training was given for $1 \mathrm{hr} / \mathrm{d}$ over a period of 4 weeks, resulting in $20 \mathrm{~d}$ of training for each subject. Subjects participated in a pretraining session the day before the training began, and a post-training session was scheduled on the day after the last training session. EEG was measured during pretraining, post-training, during every session for the first week, and during every second session thereafter. The experimental design is presented schematically in Figure 1.

During pretraining, training, and post-training sessions, subjects were seated in a comfortable chair with both arms and hands on armrests. Embedded in each armrest were two pneumatically driven stimulation devices; the centers of the volar surface of the distal phalanx of D1 and D5 were positioned above them. Each stimulation unit consisted of a pad with four holes containing pins arranged in a diamond $6 \mathrm{~mm}$ on a side. The stimulation patterns are presented in Figure 2. Only the left hand was trained. During training, three of the four pins were activated, forming an arrow-like pattern pointing to either the left or the right. For $80 \%$ of the trials, D1 and D5 were stimulated simultaneously, half with arrows pointing to the left and half with arrows pointing to the right. For another $10 \%$ of the trials, the stimuli were applied to D1 and for the remaining $10 \%$ to D5; half of the arrows pointed to the left and half to the right. The stimulation of either D1 or D5 alone served as test trials, making it possible to assess changes in the spatial location of the cortical representation of the fingers. The stimulus duration was $50 \mathrm{msec}$, and the interstimulus interval varied randomly between 1875 and $2125 \mathrm{msec}$ (mean of $2000 \mathrm{msec}$ ). Stimulation was delivered in three blocks of $22 \mathrm{~min}$ each with $5 \mathrm{~min}$ rest intervals between blocks. Each block consisted of 650 stimuli. Stimulus conditions were presented in a pseudorandom order adjusted so that left- and right-facing patterns were applied with equal frequency in each block. Subjects were asked to discriminate the direction of the stimulus pattern and to respond by pressing either a left or a right button with their right (untrained) hand corresponding to the direction of the pattern. A video screen provided feedback on whether the preceding response was correct $(+)$ or incorrect $(-)$; the percentage of correct responses on previous trials of the block was shown directly above the individual preceding-trial feedback. Subjects were instructed to achieve as high a hit rate as possible to direct and maintain attention to the stimulation, thereby making the task behaviorally relevant. Hit rate and reaction time in response to the stimulation was recorded and subjected to ANOVA with the within-factors stimulation (D1 vs D5) and training period (first, second, and third) (Fig. 1).

During the pretraining and post-training sessions, only the two pins along the finger axis were activated. Thus, the stimulus configuration had no directionality and the subject was not called on to make a response.

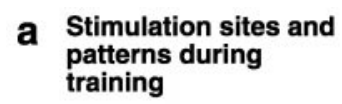
b Stimulation sites and patterns during pre- and post- training measurements
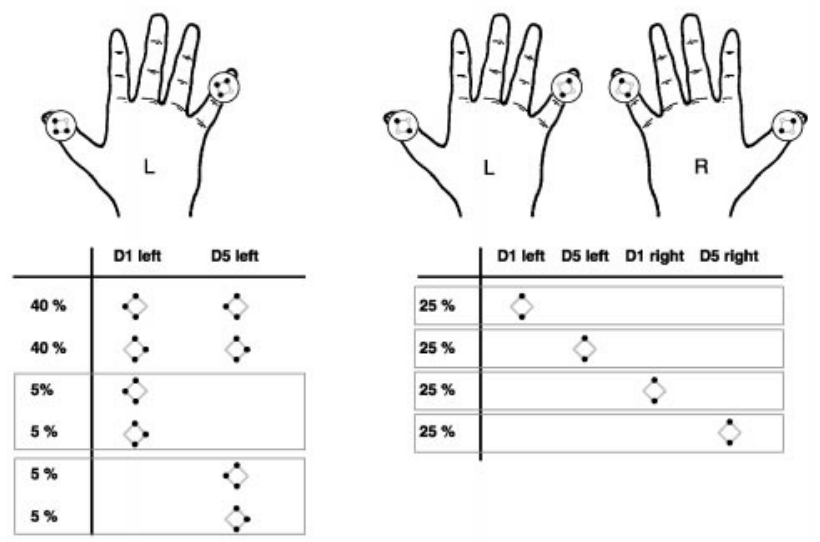

Figure 2. Stimulation sites and stimulation patterns for tactile discrimination training $(a)$, and pretraining and post-training (nondiscrimination) (b) sessions. The frequencies of the various stimulation patterns are indicated in the bottom of the figure. Conditions that were entered separately in the neuroelectric source localization are framed by gray rectangles. The rectangles in $a$ contain two stimulation patterns each, indicating that the two trial types were collapsed for the source analysis.

This might be characterized as a passive stimulation mode. The stimulus was delivered to either D1 or D5 in pseudorandom order, with each site being stimulated an equal number of times during the session. There were again three blocks of 650 stimuli with a 5 min rest period between blocks.

Mislocalization test. The distribution of mislocalizations to digits other than the one stimulated was tested with von Frey hairs using a fivealternative, forced-choice detection procedure with sight of the hands occluded. On each trial, the tip of the nylon filament of a von Frey hair was applied manually to the skin, and pressure was exerted on its attached rod until the filament bent. The von Frey hairs were applied to the center of the volar surface of the first phalanx of each digit on each trial for $\sim 1 \mathrm{sec}$. This was followed by a $7 \mathrm{sec}$ interval in which the subject had to name the stimulated finger. The hands were tested separately, and each finger was stimulated 20 times in random order. To ensure a comparable number of mislocalizations from stimulation of each finger, a tracking procedure was used that adjusted the stimulation intensity to the threshold of each finger for correct localization. Each correct response was followed by a lower stimulation intensity and each false response by a higher intensity for the succeeding stimulation of that finger. This procedure resulted in there being an $\sim 50 \%$ chance of false responses (i.e., mislocalizations) for stimulus presentations to each finger. The rate of mislocalizations is reported only for stimulation of either $\mathrm{D} 1$ or D5. In the case of D1 stimulation, D2 was designated the first neighbor and D5 the fourth neighbor. Conversely, when D5 was the stimulated digit, D4 was designated the first neighbor finger and D1 the fourth neighbor. By using this schema for digit characterization, it was possible to combine the mislocalization data for stimulation of the two digits and to analyze mislocalization in terms of the distance from a stimulated finger. ANOVA factors were week (first vs fourth), neighboring digit (first vs fourth), and hand (trained vs untrained).

$E E G$. Neuroelectric source imaging was used to determine the location of the center of cortical responsivity to stimulation of D1 and D5. Somatosensory evoked potentials were obtained from 128 electrodes referenced to $\mathrm{Cz}$ (vertex electrode) using EGIS-Geodesic Sensor Net and Net amplifiers (Electrical Geodesics Inc., Eugene, OR). The average distance between electrodes was $\sim 3 \mathrm{~cm}$. All signals were sampled at a rate of $500 \mathrm{~Hz}$ with a band pass from 0.1 to $200 \mathrm{~Hz}$. The continuous data stream was cut into sweeps of $1050 \mathrm{msec}$ with a baseline of $50 \mathrm{msec}$. All 128 channels were scanned on a single-trial basis for artifacts, defined as amplitudes exceeding the bandwidth of the analog-to-digital converter or activity changes larger than 10 times the SD of the signal. Channels with $>25 \%$ bad trials were excluded from analysis, as were trials that showed artifacts on any of the remaining channels (median rejection rate of $15 \%)$. To achieve a complete data set, excluded channels were replaced 

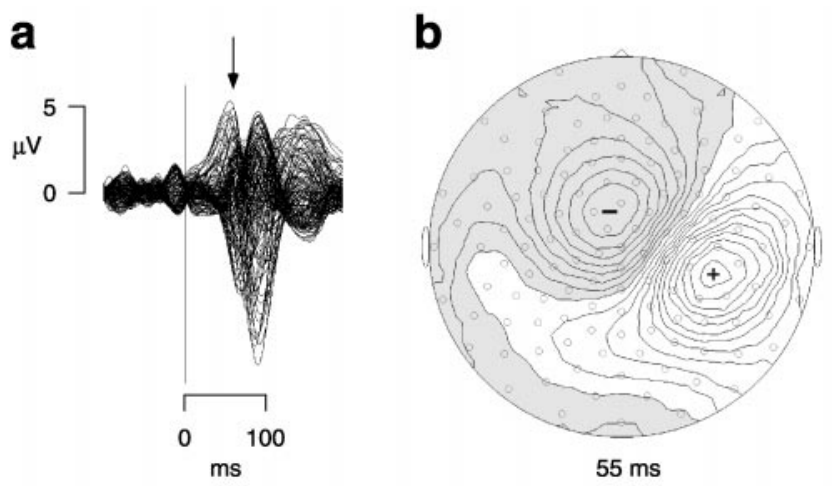

Figure 3. A superposition of an evoked response to stimulation of D5 from all 128 channels is illustrated for a single subject $(a)$. Stimulus onset is at 0 msec. The arrow marks the SI peak used for source analysis; its topography is shown on the right $(b)$. (The subsequent later peaks receive contributions from SII and also posterior parietal cortex, making source modeling more difficult).

by means of a spline interpolation (Junghöfer et al., 1997). Stimulation conditions were then averaged across trials. To increase the signal-tonoise ratio, four to five training sessions were pooled. All single-subject EEG responses for stimulation of D1 and D5 individually were submitted to a source analysis using the BESA 2.2 program (MEGIS Software $\mathrm{GmbH}$, Munich, Germany) (Scherg, 1990). The first prominent activity in the range of 40-80 msec (Elbert et al., 1994, 1995; Flor et al., 1995) was fitted with a single equivalent dipole model. The positions of the 129 electrodes (including the $\mathrm{Cz}$ reference) were digitized (Polhemus) and projected onto the BESA spherical head model. The coordinate system used had its origin in the center of the sphere, its $z$-axis pointing toward $\mathrm{Cz}$, its $x$-axis oriented in the mediolateral direction, and its $y$-axis pointed in the anteroposterior direction. In polar coordinates, the polar angle $(\theta)$ describes the location of a given point on the postcentral gyrus when the azimuth is set close to $0^{\circ}$ (see Fig. 5). Source locations anterior or posterior to the central sulcus would result in variations of the azimuth $\phi$ different from zero.

Dipole parameters from training sessions were subjected to an ANOVA with the factors digit (D1 vs D5) and training period (first, second, and third). The ANOVA for the pretraining and post-training sessions comprised the factors digit (D1 vs D5) and measurement (pretraining vs post-training).

\section{RESULTS}

\section{Neuroelectric source localization}

A typical example of the evoked response is presented in Figure 3. In each case, a first prominent peak could be easily identified in the latency region of 50-60 msec. Its scalp topography revealed a dipolar pattern with polarity reversal in the region over the central sulcus, contralateral to the stimulated side (Fig. 3b). Its source location was well modeled by a single dipole that was located in the region of the central sulcus as verified by overlay onto the individual magnetic resonance image.

As a result of training on the discrimination task, the threedimensional Euclidean distance between the cortical representations of D1 and D5 contralateral to the trained hand increased substantially $\left(F_{(2,8)}=7.90 ; p<0.05 ; \epsilon=0.905\right)$ during the discrimination task. Figure 4 indicates that a measure of the distance in the mediolateral dimension (primarily along the surface of the postcentral gyrus) between the locations (polar angle, $\Delta \theta$ ) went from a mean $\pm \mathrm{SE}$ of $1.5 \pm 3.3^{\circ}$ during the first period of training to a mean of $7.4 \pm 2.8^{\circ}$ during the final period of training. There was an increase in dipole strength across training periods for D5 $\left(F_{(2,8)}=6.0 ; p=0.05 ; \epsilon=0.88\right)$. No significant changes in the orientation of the dipole were observed across the training, and there were no changes in location along the anteroposterior axis. a

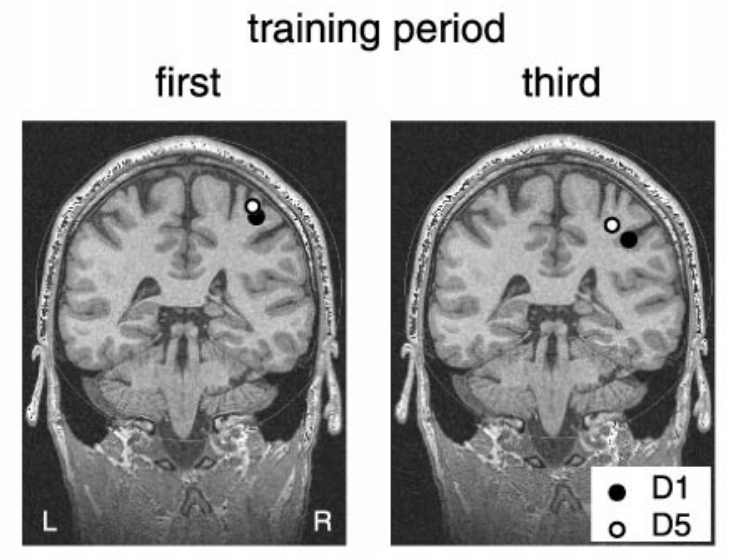

b

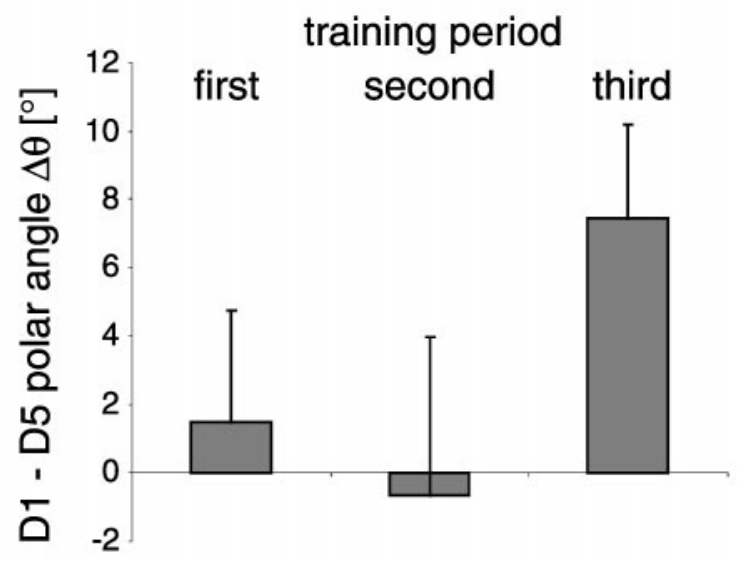

Figure 4. Neuroelectric source imaging data for tactile discrimination training sessions. $a$, Coronal section through the postcentral gyrus (somatosensory cortex) of one experimental subject showing the increase in polar angle between the cortical representations of the first and fifth digit from the first period of training (left) to the last period of training (right). $b$, Mean polar angle for experimental subjects (group data) between the D1 and D5 dipoles during the first, second, and last period of discrimination training.

The opposite effect was observed in response to passive stimulation of D1 and D5 separately (Fig. 5). There was a decrease in polar angle from the pretraining session to the post-training session of $11.8^{\circ}$, going from $10.3 \pm 3.3^{\circ}$ pretraining to $-1.5 \pm 2.1^{\circ}$ post-training $\left(F_{(1,4)}=8.1 ; p<0.05\right)$. The dipoles for the fingers of the untrained hand were not significantly different from one another between the pretraining and post-training periods.

\section{Mislocalization test}

When tactile stimulation is delivered at near-threshold values, there is a tendency for subjects to make errors in identifying which finger was touched. The greatest number of mislocalized attributions are usually to the digit closest to the digit being stimulated (first neighbor digit) and seldom to the most distant digit (fourth neighbor when the stimulated digit is to either D1 or D5). Figure 6 indicates that, in 18 right-handed control subjects who received no training, there was a decrease in mislocalizations from the first neighbor finger to the fourth neighbor finger for both the left and the right hands $\left(F_{(1,17)}=121.4 ; p<0.0001\right)$. A similar phenomenon was observed: (1) in the untrained hand in experimental subjects during both the first week of training and during the fourth week of training $\left(F_{(1,3)}=15.6 ; p<0.05\right)$, and (2) in the trained hand during the first week of training $\left(F_{(1,3)}=\right.$ 


\section{a}
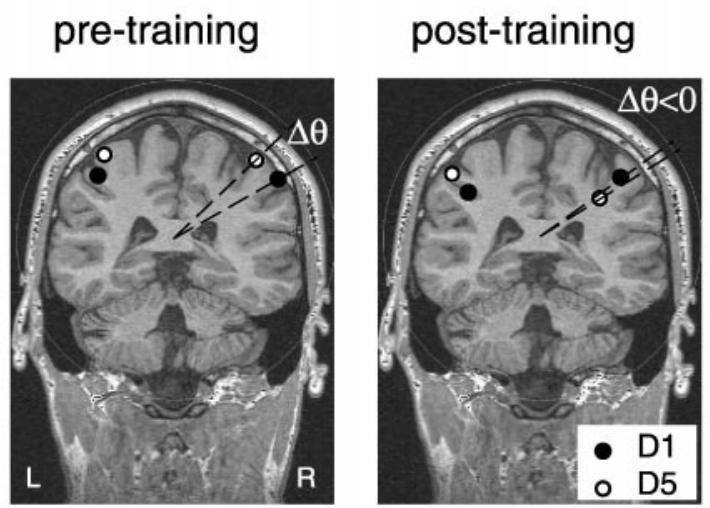

b

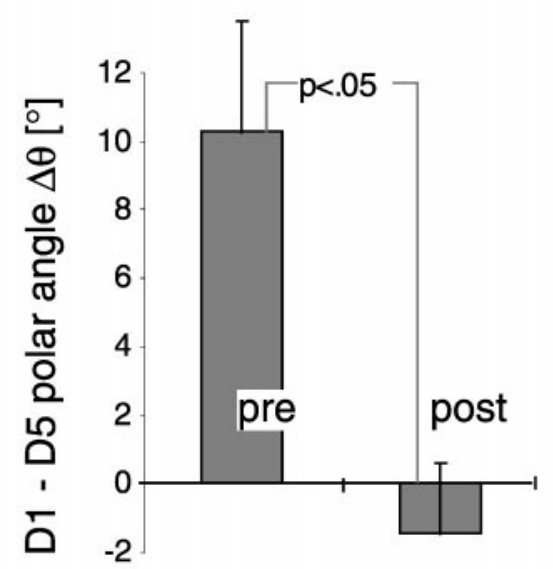

Figure 5. Neuroelectric source imaging data for passive tactile stimulation sessions. $a$, Coronal section through the postcentral gyrus of one experimental subject showing the decrease in polar angle between the cortical representations of the first and fifth digit from the pretraining to the post-training sessions. $b$, Mean polar angle for experimental subjects (group data) between the D1 and D5 dipoles for the pretraining and post-training passive stimulation sessions.

8.5; $p<0.06)$. However, during the fourth week of training, there was a striking change so that mislocalizations for the trained hand were as frequently attributed to the fourth neighbor as to the first neighbor finger. The difference was not significant for the trained hand at the end of training $(F=0.001)$.

The increase in the number of mislocalizations attributed to the fourth neighbor finger compared with the first neighbor finger at the end of the training was highly correlated with the decrease in distance between cortical D1 and D5 representations in response to passive stimulation that also occurred as a result of training $(r=-0.99 ; p<0.0001)$. Before training, this correlation was not significant.

\section{Discrimination task}

Reaction time in the discrimination procedure did decrease with training $\left(F_{(2,8)}=42.5 ; p<0.001 ; \epsilon=0.869\right)$, but it did not decrease differentially for the different types of stimulation. The correct response (hit) rate for the directional discrimination task was $81.1 \%$ in the first period of training (week 1), $92.0 \%$ in the second period of training (week 2 and first half of week 3 ), and $91.7 \%$ in the third period of training (second half of week 3 and week 4) $\left(F_{(2,8)}=11.3 ; p<0.05 ; \epsilon=0.641\right)$.
Trained subjects

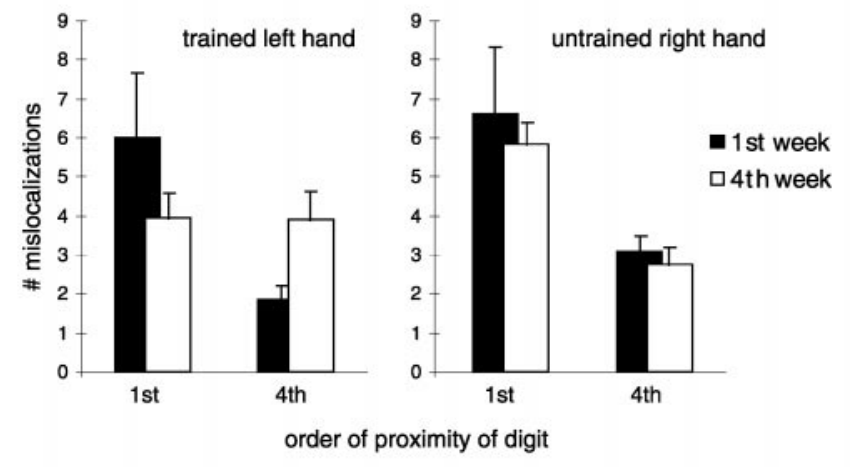

Controls

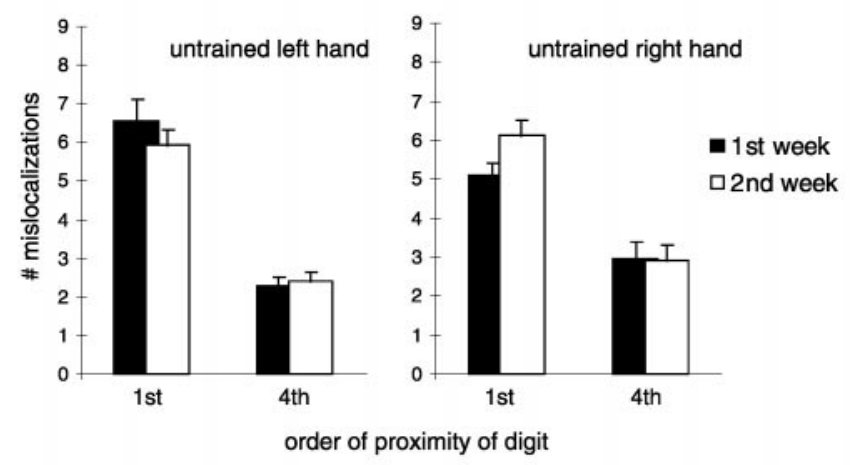

Figure 6. Number of mislocalizations attributed to the first and fourth neighbor fingers during the first week of training and the final week of training for the trained left hands (top row) and the untrained right hands of the experimental subjects and for the left and right untrained hands of 18 additional control subjects (bottom row).

\section{DISCUSSION}

Task performance was not error free, indicating that it required attention and benefited from learning. The task therefore qualifies as providing the type of behaviorally relevant stimulation found to produce use-dependent cortical reorganization (Jenkins et al., 1990; Elbert et al., 1995; Buonomano and Merzenich, 1998; Sterr et al., 1998 a,b). Previous research has shown that repetitive, behaviorally relevant stimulation produces an expansion in the topographic extent of the area occupied by the representation of a body part in somatosensory cortex. In contrast, repetitive stimulation of two body sites simultaneously has been found to yield a fused (Wang et al., 1995) or disarranged (Sterr et al., 1998a,b) representation of those portions of the body. In this experiment, both effects were found to occur as a consequence of the prolonged repetitive application of a single type of task-relevant stimulation; they were, however, elicited by different types of stimulus conditions after training. When the situation required that the subjects made a discrimination involving a certain degree of difficulty (as indicated by the less than perfect hit rate), the polar angle between cortical D1 and D5 was increased. This suggests that their representation was expanded or that at each training stage different regions of a more general map became activated. When the stimulation during assessment was passive, involving no discriminative task, the distance between the cortical representations of the two digits was decreased and their topographic order was disarranged. We may conclude that the 
discrimination training tended to isolate pools of neurons activated by D1 or D5 stimuli to the low-threshold territories in primary somatosensory cortex (SI), whereas the passive stimuli tended to enlarge the territories toward each other, possibly resulting in an overlap.

Studies with intracranial electrodes suggest that primary somatosensory area $3 \mathrm{~b}$ and area 1 contribute to the peak in the evoked response currently examined (Baumgartner et al., 1993). If the ratio of the contributions from these two regions would have changed during the training or if activity from other areas, such as areas 2 or $3 \mathrm{a}$, would have altered the response, then a change in the orientation of the equivalent current dipole would have been the consequence. The training did not affect the dipole orientation nor did it change the localization along the anteroposterior axis (azimuth). Therefore, it is unlikely that differential activation of digit representations in different cortical areas could have produced the observed effects.

The accuracy of source localizations based on high-resolution EEG depends on the signal-to-noise ratio, the number of electrodes, and the location of the source. Superficial sources can be more precisely located than those at deeper locations. Source modeling using simulated data indicate that with as few as 60 EEG channels, the accuracy of dipole locations within the somatosensory cortex is already within a range of 3-5 mm (95\% confidence interval), i.e., below the variations currently observed (Braun et al., 1997).

The very high correlation between shifts in the location of the cortical representation of the fingers and the perceptual phenomena are striking. The shift in mislocalizations so that they were equally distributed between the finger farthest from and closest to the stimulated finger may be a perceptual consequence of the fused and disordered cortical digital representations.

The different task-specific cortical effects that were observed were elicited by two different types of stimulus conditions during assessment. Previous investigators had not used a dual discrimination approach such as the one used here; they could therefore not have observed the phenomenon found in this experiment. It will be challenging to derive such evidence in animal studies, because maps would need to be determined in an awake, behaving animal to demonstrate the context specificity.

However, a number of well known studies have shown that, in the visual system, several different types of discrimination can be processed in the same small area of occipital cortex. A similar phenomenon has been demonstrated recently by Ungerleider, Karni, and coworkers (Karni et al., 1995; Ungerleider, 1995) for the motor system. Using functional magnetic resonance imaging, they showed that two different keyboard tasks using the same musculature and differing only in the pattern of activation of the muscles were processed differently in primary motor cortex. In the present experiment, a correlative phenomenon was demonstrated for the somatosensory system. It was found that a single type of prolonged repetitive simultaneous stimulation of two digits produced two different and opposite use-dependent effects on the spatial relationship of the cortical representations of the digits, depending on the nature of the discrimination condition used during neuroimaging sessions. One possibility is that differential activation of a general map may mimic multiple maps sharing the same region of somatosensory cortex that are specific to different modes of stimulus processing within the somatosensory modality. Alternatively, it seems possible that multiple maps, each dependent on context, could coexist at the same time in the same cortex, but evidence for this would require rapid switching back and forth from one context to the other while recording. Without such evidence, it is further possible that different maps may exist at different times in each individual.

\section{REFERENCES}

Allard T, Clark SA, Jenkins WM, Merzenich MM (1991) Reorganization of somatosensory area $3 \mathrm{~b}$ representations in adult owl monkeys after digital syndactyly. J Neurophysiol 66:1048-1058.

Baumgartner C, Doppelbauer A, Sutherling WW, Lindinger G, Levesque MF, Aull S, Zeitlhofer J, Deecke L (1993) Somatotopy of human somatosensory cortex as studied in scalp EEG. Electroencephalogr Clin Neurophysiol 88:271-279.

Braun C, Kaiser S, Kincses WE, Elbert T (1997) Confidence interval of single dipole locations based on EEG data. Brain Topogr 10:31-39.

Buonomano DV, Merzenich MM (1998) Cortical plasticity: from synapses to maps. Annu Rev Neurosci 21:149-186.

Clark SA, Allard T, Jenkins WM, Merzenich MM (1988) Receptive fields in the body surface map in adult cortex defined by temporally correlated inputs. Nature 332:444-445.

Elbert T, Flor H, Birbaumer N, Knecht S, Hampson S, Larbig W, Taub E (1994) Extensive reorganization of the somatosensory cortex in adult humans after nervous system injury. NeuroReport 5:2593-2597.

Elbert T, Pantev C, Wienbruch C, Rockstroh B, Taub E (1995) Increased cortical representation of the fingers of the left hand in string players. Science 270:305-307.

Elbert T, Sterr A, Flor H, Rockstroh B, Knecht S, Pantev C, Wienbruch C, Taub E (1997) Input-increase and input-decrease types of cortical reorganization after upper extremity amputation in humans. Exp Brain Res 117:161-164.

Flor H, Elbert T, Knecht S, Wienbruch C, Pantev C, Birbaumer N, Larbig W, Taub E (1995) Phantom-limb pain as a perceptual correlate of cortical reorganization following arm amputation. Nature 375:482-484.

Jenkins WM, Merzenich MM, Ochs MT, Allard T, Guic-Robles E (1990) Functional reorganization of primary somatosensory cortex in adult owl monkeys after behaviorally controlled tactile stimulation. J Neurophysiol 63:82-104.

Junghöfer M, Elbert T, Leiderer P, Berg P, Rockstroh B (1997) Mapping EEG-Potentials on the surface of the brain: a strategy for uncovering cortical sources. Brain Topogr 9:203-217.

Kaas JH (1995) The reorganization of sensory and motor maps in adult mammals. In: The cognitive neurosciences (Gazzaniga MS, ed), pp 51-71. Cambridge, MA: MIT.

Karni A, Meyer G, Jezzard P, Adams MM, Turner R, Ungerleider LG (1995) Functional MRI evidence for adult motor cortex plasticity during motor skill learning. Nature 377:155-158.

Merzenich MM, Nelson RJ, Stryker MP, Cynader MS, Schoppmann A, Zook JM (1984) Somatosensory cortical map changes following digit amputation in adult monkeys. J Comp Neurol 224:591-605.

Mogilner A, Grossman JA, Ribary U, Joliot M, Volkmann J, Rapaport D, Beasley R W, Llinas RR (1993) Somatosensory cortical plasticity in adult humans revealed by magnetoencephalography. Proc Natl Acad Sci USA 90:3593-3597.

Mühlnickel W, Elbert T, Taub E, Flor H (1998) Reorganization of auditory cortex in tinnitus. Proc Natl Acad Sci USA 95:10340-10343.

Oldfield RC (1971) The assessment and analysis of handedness: the Edinburgh inventory. Neuropsychologia 9:97-113.

Recanzone GH, Merzenich MM, Jenkins WM, Grajski KA, Dinse HR (1992) Changes in the distributed temporal response properties of SI cortical neurons reflect improvements in performance on a temporally based tactile discrimination task. J Neurophysiol 67:1031-1056.

Scherg M (1990) Fundamentals of dipole source potential analysis. In: Auditory evoked magnetic fields and electric potentials (Grandori F, Hoke M, Romani GL, eds), pp 40-69. Basel: Karger.

Sterr A, Müller MM, Elbert T, Rockstroh B, Pantev C, Taub E (1998a) Changed perceptions in braille readers. Nature 391:134-135.

Sterr A, Müller MM, Elbert T, Rockstroh B, Pantev C, Taub E (1998b) Perceptual correlates of use-dependent changes in cortical representation of the fingers in blind braille readers. J Neurosci 18:4417-4423.

Ungerleider LG (1995) Functional brain imaging studies of cortical mechanisms for memory. Science 270:769-775.

Wang X, Merzenich MM, Sameshima K, Jenkins WM (1995) Remodeling of hand representation in adult cortex determined by timing of tactile stimulation. Nature 378:71-75. 